\title{
Class Management Profiles of Secondary School Teachers
}

\author{
Muhsin Hazar \\ Gazi University, Turkey \\ Malik Beyleroglu \\ Sakarya University, Turkey \\ Sakir Bezci \\ Karabük University, Turkey \\ Hacer Ozge Baydar \\ Emre Ozan Tingaz \\ Gazi University, Turkey
}

Doi: 10.19044/esj.2017.v13n34p381 URL:http://dx.doi.org/10.19044/esj.2017.v13n34p381

\begin{abstract}
The purpose of this research is to examine the class management profile of secondary school branch teachers working in certain province of Turkey, using some variables. The research group consists of 152 teachers (69 female and 83 male) in the various field of Mathematics (29), Turkish (46), Religion Culture (23), English (31), and Physical Education and Sports (23). The "Classroom Management Profile Scale (SYPÖ)" developed by Kris (1996) and adapted to Turkish by Ekici (2004) was used as the data collection tool in the research. The research was conducted based on both the general and the relational screening models. The SPSS 20.0 package program was used in the analysis of the data. Both $\mathrm{T}$ test and one-way ANOVA were used in independent groups to determine whether the gender and subscale of the scale were significantly different according to gender, age, occupation year and field. The results of the research proved that, when the scores of both scales were calculated, the grade management profiles of the teachers were not statistically different according to the considered variables.
\end{abstract}

Keywords: Classroom Management, Classroom Management Profile, Secondary Education

\section{Introduction}

One of the most important factors that affect teaching in a learning environment is the teacher's class management profile. The teacher's goal is 
to effect a change of behavior in the students through the class management methods applied, and try to ensure that a sufficient level of motivation is formed in them. Teachers' behavior patterns in the communication process constitute their class management professions (Aluçdibi \& Ekici, 2012).

However, the aim of all activities carried out in the classroom environment is to enable the student to make effective learning. However, many factors can positively or adversely affect this objective - one of the most important being the teacher's behavior. The positive effect of the teacher on the student will be ensured by the positive communication process between the teacher and the student (Ekici, 2004). The more consistent the communication between teachers and students in the classroom is, the more they are willing to learn (Brown, 2005).

Classroom management includes: the provision of motivation for students to establish an appropriate environment for learning in class, to organize physical arrangements, the flow of teaching and time management, to organize relations in a classroom environment within certain rules, and to ensure effective coordination between the teacher and the student (Saritaş, 2003).

A value that is closely related to the students' academic achievement and behavior in the school climate is the teacher's classroom management profile (Kurt, 2013). The factors that arise, especially from the teaching resulting to a boost or a decline in the academic success of the students, are the most important issues in the researches carried out in the field of education and training. In this context, it is important to examine the classroom management professions of the prospective teachers. For this reason, it is thought that this research will contribute to the field.

The purpose of this research is to investigate or examine the class management professions of the secondary school branch teachers who work in regards to their age, gender, year of profession year, and other variables.

\section{Method}

\section{Research Group}

The research group consists of 152 teachers, 69 of whom are female and 83 of whom are male. Among them, 29 are in the field of Mathematics, 46 Turkish, 23 Religion, 31 English, and 23 are in Physical Education and Sports branches in Ankara, Zonguldak, and Gaziantep.

\section{Research Model}

The research was based on general screening models and relational screening model.

Screening models are research approaches that aim to describe the past or present as if there is an existing situation. Relational screening 
models are research models aimed at determining the presence and/or degree of exchange between two or more variables (Karasar, 2000).

\section{Data Collection Tools}

The "Classroom Management Profile Scale (SYPÖ)" developed by Kris (1996) and adapted to Turkish by Ekici (2004) was used in the research. The scale, which is composed of a total of 12 items for Authoritative Classroom Management Profile, Appreciating Classroom Management Profile, Unattached Classroom Management Profile and Indulgence Classroom Management Profile type, allows for individual evaluation.

\section{Analysis of Data}

In the analysis of the data, SPSS 20.0 package program was used. Both $\mathrm{T}$ test and one-way analysis of variance were used in independent groups to determine whether the gender and subscale of the scale were significantly different according to gender, age, occupation year and field.

\section{Results}

1. Distribution of secondary school branch teachers by division, age, occupational year, and gender variables are given in Tables 1, 2, 3, and 4.

Table 1.

\begin{tabular}{|l|l|l|}
\hline Branch & \multicolumn{1}{|c|}{ Frequency } & Percent (\%) \\
\hline Math Teacher & 29 & 19,1 \\
\hline Turkish Teacher & 46 & 30,3 \\
\hline Physical Education Teacher & 23 & 15,1 \\
\hline English Teacher & 31 & 20,4 \\
\hline Religious Culture Teacher & 23 & 15,1 \\
\hline Total & 152 & 100,0 \\
\hline
\end{tabular}

Table 2.

\begin{tabular}{|l|l|l|}
\hline Occupational Year & Frequency & Percent $(\%)$ \\
\hline 1 year and below & 27 & 17,8 \\
\hline 2-4 years & 55 & 36,2 \\
\hline 5-7 years & 23 & 15,1 \\
\hline 8-10 years & 10 & 6,6 \\
\hline 11 years and over & 37 & 24,3 \\
\hline Total & 152 & 100,0 \\
\hline
\end{tabular}

Table 3.

\begin{tabular}{|l|l|l|}
\hline Gender & Frequency & Percent (\%) \\
\hline Female & 69 & 45,4 \\
\hline Male & 83 & 54,6 \\
\hline Total & 152 & 100,0 \\
\hline
\end{tabular}


Table 4.

\begin{tabular}{|l|l|l|}
\hline Age & Frequency & Percent $(\%)$ \\
\hline 24 years and under & 26 & 17,1 \\
\hline 25-29 years & 49 & 32,2 \\
\hline 30-34 years & 39 & 25,7 \\
\hline 35-39 years & 24 & 15,8 \\
\hline 40 years and over & 14 & 9,2 \\
\hline Total & 152 & 100,0 \\
\hline
\end{tabular}

2. Are there differences in scores from subscale to subscale scores?

Table 5.

\begin{tabular}{|c|c|c|c|c|c|c|}
\hline & Branch & $\mathrm{n}$ & Order Average & $\begin{array}{l}\text { Degree of } \\
\text { Freedom }\end{array}$ & $\chi^{2}$ & $\mathrm{P}$ \\
\hline \multirow{5}{*}{$\begin{array}{l}\text { Authoritative } \\
\text { Classroom } \\
\text { Management } \\
\text { Profile }\end{array}$} & $\begin{array}{l}\text { Math } \\
\text { Teacher }\end{array}$ & 29 & 69,72 & \multirow[t]{5}{*}{4} & \multirow[t]{5}{*}{2,959} & \multirow[t]{5}{*}{0,565} \\
\hline & $\begin{array}{l}\text { Turkish } \\
\text { Teacher }\end{array}$ & 46 & 78,38 & & & \\
\hline & $\begin{array}{l}\text { Physical } \\
\text { Education } \\
\text { Teacher }\end{array}$ & 23 & 88,74 & & & \\
\hline & $\begin{array}{l}\text { English } \\
\text { Teacher }\end{array}$ & 31 & 72,21 & & & \\
\hline & $\begin{array}{l}\text { Religious } \\
\text { Culture } \\
\text { Teacher } \\
\end{array}$ & 23 & 74,83 & & & \\
\hline \multirow{5}{*}{$\begin{array}{l}\text { Appreciated } \\
\text { Classroom } \\
\text { Management } \\
\text { Profile }\end{array}$} & $\begin{array}{l}\text { Math } \\
\text { Teacher }\end{array}$ & 29 & 87,53 & \multirow[t]{5}{*}{4} & \multirow[t]{5}{*}{8,194} & \multirow[t]{5}{*}{0,085} \\
\hline & $\begin{array}{l}\text { Turkish } \\
\text { Teacher }\end{array}$ & 46 & 80,95 & & & \\
\hline & $\begin{array}{l}\text { Physical } \\
\text { Education } \\
\text { Teacher }\end{array}$ & 23 & 58,09 & & & \\
\hline & $\begin{array}{l}\text { English } \\
\text { Teacher }\end{array}$ & 31 & 81,08 & & & \\
\hline & $\begin{array}{l}\text { Religious } \\
\text { Culture } \\
\text { Teacher }\end{array}$ & 23 & 65,93 & & & \\
\hline \multirow{5}{*}{$\begin{array}{l}\text { Free } \\
\text { Classroom } \\
\text { Management } \\
\text { Profile }\end{array}$} & $\begin{array}{l}\text { Math } \\
\text { Teacher }\end{array}$ & 29 & 66,22 & \multirow[t]{5}{*}{4} & \multirow[t]{5}{*}{5,025} & \multirow[t]{5}{*}{0,285} \\
\hline & $\begin{array}{l}\text { Turkish } \\
\text { Teacher }\end{array}$ & 46 & 86,98 & & & \\
\hline & $\begin{array}{l}\text { Physical } \\
\text { Education } \\
\text { Teacher } \\
\end{array}$ & 23 & 69,89 & & & \\
\hline & $\begin{array}{l}\text { English } \\
\text { Teacher }\end{array}$ & 31 & 77,92 & & & \\
\hline & $\begin{array}{l}\text { Religious } \\
\text { Culture }\end{array}$ & 23 & 73,20 & & & \\
\hline
\end{tabular}




\begin{tabular}{|c|c|c|c|c|c|c|}
\hline & Teacher & & & & & \\
\hline \multirow{5}{*}{$\begin{array}{l}\text { Unsusceptible } \\
\text { Classroom } \\
\text { Management } \\
\text { Profile }\end{array}$} & $\begin{array}{l}\text { Math } \\
\text { Teacher }\end{array}$ & 29 & 74,16 & \multirow[t]{5}{*}{4} & \multirow[t]{5}{*}{5,354} & \multirow[t]{5}{*}{0,253} \\
\hline & $\begin{array}{l}\text { Turkish } \\
\text { Teacher }\end{array}$ & 46 & 79,38 & & & \\
\hline & $\begin{array}{l}\text { Physical } \\
\text { Education } \\
\text { Teacher }\end{array}$ & 23 & 92,46 & & & \\
\hline & $\begin{array}{l}\text { English } \\
\text { Teacher }\end{array}$ & 31 & 70,24 & & & \\
\hline & $\begin{array}{l}\text { Religious } \\
\text { Culture } \\
\text { Teacher }\end{array}$ & 23 & 66,17 & & & \\
\hline \multirow{5}{*}{ Total } & $\begin{array}{l}\text { Math } \\
\text { Teacher }\end{array}$ & 29 & 75,29 & \multirow[t]{5}{*}{4} & \multirow[t]{5}{*}{3,858} & \multirow[t]{5}{*}{0,426} \\
\hline & $\begin{array}{l}\text { Turkish } \\
\text { Teacher }\end{array}$ & 46 & 84,17 & & & \\
\hline & $\begin{array}{l}\text { Physical } \\
\text { Education } \\
\text { Teacher } \\
\end{array}$ & 23 & 76,57 & & & \\
\hline & $\begin{array}{l}\text { English } \\
\text { Teacher }\end{array}$ & 31 & 76,77 & & & \\
\hline & $\begin{array}{l}\text { Religious } \\
\text { Culture } \\
\text { Teacher }\end{array}$ & 23 & 62,24 & & & \\
\hline
\end{tabular}

There is no statistically significant difference between the total scores from the subscale of Authoritarian Classroom Management Profile $\left(\chi^{2}(\mathrm{sd}=4\right.$, $\mathrm{n}=152)=2,959 ; \mathrm{p}=0,565>0,05$ (Kruskal Wallis Test).

There is no statistically significant difference between the total scores from the subscale of Appraisal Classroom Management Profile $\left(\chi^{2}(\mathrm{sd}=4\right.$, $\mathrm{n}=152)=8,194 ; \mathrm{p}=0,085>0,05$ (Kruskal Wallis Test).

There is no statistically significant difference between the total scores from the sub-scale of the Free Classroom Management Profile $\left(\chi^{2}(\mathrm{sd}=4, \mathrm{n}=152)=5,025 ; \mathrm{p}=0,285>0,05\right.$ (Kruskal Wallis Test).

There is no statistically significant difference between the total scores from the subscale of the Classroom Management Profile according to the situation $\left(\chi^{2}(\mathrm{sd}=4, \mathrm{n}=152)=5,354 ; \mathrm{p}=0,253>0,05\right.$ (Kruskal Wallis Test).

There is no statistically significant difference between the total scores of the scale $\left(\chi^{2}(\mathrm{sd}=4, \mathrm{n}=152)=3,858 ; \mathrm{p}=0,426>0,05\right.$ (Kruskal Wallis Test). 


\section{Are the scores according to age different from the scale and subscales?}

Table 6.

\begin{tabular}{|c|c|c|c|c|c|c|}
\hline & Age & $\mathrm{N}$ & Order Average & $\begin{array}{l}\text { Degree of } \\
\text { Freedom }\end{array}$ & $\chi^{2}$ & $P$ \\
\hline \multirow{5}{*}{$\begin{array}{l}\text { Authoritative } \\
\text { Classroom } \\
\text { Management } \\
\text { Profile }\end{array}$} & $\begin{array}{l}24 \quad \text { years } \\
\text { and under }\end{array}$ & 26 & 64,37 & \multirow[t]{5}{*}{4} & \multirow[t]{5}{*}{6,976} & \multirow[t]{5}{*}{0,137} \\
\hline & 25-29 years & 49 & 79,14 & & & \\
\hline & $30-34$ years & 39 & 88,32 & & & \\
\hline & $35-39$ years & 24 & 64,42 & & & \\
\hline & 40 years & 14 & 77,57 & & & \\
\hline \multirow{5}{*}{$\begin{array}{l}\text { Appreciated } \\
\text { Classroom } \\
\text { Management } \\
\text { Profile }\end{array}$} & $\begin{array}{l}24 \text { years } \\
\text { and under }\end{array}$ & 26 & 62,46 & \multirow[t]{5}{*}{4} & \multirow[t]{5}{*}{5,720} & \multirow[t]{5}{*}{0,221} \\
\hline & $25-29$ years & 49 & 73,56 & & & \\
\hline & $30-34$ years & 39 & 81,46 & & & \\
\hline & 35-39 years & 24 & 89,85 & & & \\
\hline & $\begin{array}{l}40 \text { years } \\
\text { and over }\end{array}$ & 14 & 76,14 & & & \\
\hline \multirow{5}{*}{$\begin{array}{l}\text { Free } \\
\text { Classroom } \\
\text { Management } \\
\text { Profile }\end{array}$} & $\begin{array}{l}24 \quad \text { years } \\
\text { and under }\end{array}$ & 26 & 74,12 & \multirow[t]{5}{*}{4} & \multirow[t]{5}{*}{3,022} & \multirow[t]{5}{*}{0,554} \\
\hline & $25-29$ years & 49 & 72,23 & & & \\
\hline & 30-34 years & 39 & 75,44 & & & \\
\hline & $35-39$ years & 24 & 90,29 & & & \\
\hline & $\begin{array}{l}40 \text { years } \\
\text { and over }\end{array}$ & 14 & 75,18 & & & \\
\hline \multirow{5}{*}{$\begin{array}{l}\text { Unsusceptible } \\
\text { Classroom } \\
\text { Management } \\
\text { Profile }\end{array}$} & $\begin{array}{l}24 \text { years } \\
\text { and under }\end{array}$ & 26 & 71,46 & \multirow[t]{5}{*}{4} & \multirow[t]{5}{*}{2,994} & \multirow[t]{5}{*}{0,559} \\
\hline & $25-29$ years & 49 & 80,17 & & & \\
\hline & $30-34$ years & 39 & 81,19 & & & \\
\hline & 35-39 years & 24 & 75,98 & & & \\
\hline & $\begin{array}{l}40 \text { years } \\
\text { and over }\end{array}$ & 14 & 60,82 & & & \\
\hline
\end{tabular}

There is no statistically significant difference between the total scores from the subscale of Authoritarian Classroom Management by age $(\chi 2$ $(\mathrm{sd}=4, \mathrm{n}=152)=6,976 ; \mathrm{p}=0,137>0,05$ (Kruskal Wallis Test).

There is no statistically significant difference between the total scores from the subscale of Appreciated Classroom Management Profile by Age $(\chi 2(\mathrm{sd}=4, \mathrm{n}=152)=5,720, \mathrm{p}=0.221>0.05$ (Kruskal Wallis Test).

There is no statistically significant difference between the total scores obtained from the subscale of the Adolescent Classroom Management Profile by age $(\chi 2(s d=4, n=152)=3,022, p=0,554>0,05$ (Kruskal Wallis Test). 
There is no statistically significant difference between the total scores from the subscale of the Classroom Management Profile according to age $(\chi 2(\mathrm{sd}=4, \mathrm{n}=152)=2,994, \mathrm{p}=0,559>0,05$ (Kruskal Wallis Test).

\section{Is there a difference in the scores according to the vocational year and the scores received from the Sub-Scales?}

Table 7.

\begin{tabular}{|c|c|c|c|c|c|c|}
\hline & $\begin{array}{l}\text { Occupation } \\
\text { Year }\end{array}$ & $\mathrm{n}$ & Order Average & $\begin{array}{l}\text { Degree of } \\
\text { Freedom }\end{array}$ & $\chi^{2}$ & $\mathrm{P}$ \\
\hline \multirow{5}{*}{$\begin{array}{l}\text { Appreciated } \\
\text { Classroom } \\
\text { Management } \\
\text { Profile }\end{array}$} & $\begin{array}{l}1 \text { years and } \\
\text { under }\end{array}$ & 27 & 70,31 & \multirow[t]{5}{*}{4} & \multirow[t]{5}{*}{4,414} & \multirow[t]{5}{*}{0,353} \\
\hline & $2-4$ years & 55 & 71,68 & & & \\
\hline & 5-7 years & 23 & 80,26 & & & \\
\hline & $8-10$ years & 10 & 68,10 & & & \\
\hline & $\begin{array}{l}11 \text { years } \\
\text { and over }\end{array}$ & 37 & 88,11 & & & \\
\hline \multirow{5}{*}{$\begin{array}{l}\text { Free } \\
\text { Classroom } \\
\text { Management } \\
\text { Profile }\end{array}$} & $\begin{array}{l}1 \text { years and } \\
\text { under }\end{array}$ & 27 & 84,15 & \multirow[t]{5}{*}{4} & \multirow[t]{5}{*}{4,131} & \multirow[t]{5}{*}{0,389} \\
\hline & 2-4 years & 55 & 68,65 & & & \\
\hline & 5-7 years & 23 & 87,28 & & & \\
\hline & $8-10$ years & 10 & 73,15 & & & \\
\hline & $\begin{array}{l}11 \text { years } \\
\text { and over }\end{array}$ & 37 & 76,78 & & & \\
\hline \multirow{5}{*}{$\begin{array}{l}\text { Unsusceptible } \\
\text { Classroom } \\
\text { Management } \\
\text { Profile }\end{array}$} & $\begin{array}{l}1 \text { years and } \\
\text { under }\end{array}$ & 27 & 76,28 & \multirow[t]{5}{*}{4} & \multirow[t]{5}{*}{3,223} & \multirow[t]{5}{*}{0,521} \\
\hline & $2-4$ years & 55 & 76,67 & & & \\
\hline & 5-7 years & 23 & 85,63 & & & \\
\hline & $8-10$ years & 10 & 87,75 & & & \\
\hline & $\begin{array}{l}11 \text { years } \\
\text { and over }\end{array}$ & 37 & 67,69 & & & \\
\hline
\end{tabular}

There is no statistically significant difference between the total scores obtained from the Profession Appraisal Classroom Management Profile subscale $\left(\chi^{2}(\mathrm{sd}=4, \mathrm{n}=152)=4,414 ; \mathrm{p}=0,353>0,05\right.$ (Kruskal Wallis Test).

There is no statistically significant difference between the total scores obtained from the subscale of the Adolescent Classroom Management Profession by occupational year $\left(\chi^{2}(\mathrm{sd}=4, \mathrm{n}=152)=4,131 ; \mathrm{p}=0,389>\right.$ 0,05 (Kruskal Wallis Test).

There is no statistically significant difference between the total scores obtained from the subscale of the Classroom Management Profession by occupational year $(\chi 2(s d=4, n=152)=3,223 ; \mathrm{p}=0,521>0,05$ (Kruskal Wallis Test). 
There is no statistically significant difference between the total scores (general scale) and the year of the profession $(\mathrm{F}(4,147)=0,436, \mathrm{p}=$ $0,783>0,05)$.

\section{Are there differences between scale and subscale according to sex?}

\section{Table 8.}

\begin{tabular}{|c|c|c|c|c|c|c|}
\hline & Gender & $\mathrm{n}$ & $\begin{array}{l}\text { Order } \\
\text { Average }\end{array}$ & $\begin{array}{l}\text { Rows } \\
\text { Total } \\
\end{array}$ & M.W.U. & $\mathrm{p}$ \\
\hline \multirow{2}{*}{$\begin{array}{l}\text { Authoritative } \\
\text { Classroom } \\
\text { Management } \\
\text { Profile }\end{array}$} & Female & 69 & 77,09 & 5319,00 & \multirow[t]{2}{*}{2823,000} & \multirow[t]{2}{*}{0,879} \\
\hline & Male & 83 & 76,01 & 6309,00 & & \\
\hline \multirow{2}{*}{$\begin{array}{l}\text { Appreciated } \\
\text { Classroom } \\
\text { Management } \\
\text { Profile }\end{array}$} & Female & 69 & 73,62 & 5080,00 & \multirow[t]{2}{*}{2665,000} & \multirow[t]{2}{*}{0,457} \\
\hline & Male & 83 & 78,89 & 6548,00 & & \\
\hline \multirow{2}{*}{$\begin{array}{l}\text { Free } \\
\text { Classroom } \\
\text { Management } \\
\text { Profile }\end{array}$} & Female & 69 & 75,39 & 5202,00 & \multirow[t]{2}{*}{2787,000} & \multirow[t]{2}{*}{0,774} \\
\hline & Male & 83 & 77,42 & 6426,00 & & \\
\hline \multirow{2}{*}{$\begin{array}{l}\text { Unsusceptible } \\
\text { Classroom } \\
\text { Management } \\
\text { Profile }\end{array}$} & Female & 69 & 75,70 & 5223,00 & \multirow[t]{2}{*}{2808,000} & \multirow[t]{2}{*}{0,835} \\
\hline & Male & 83 & 77,17 & 6405,00 & & \\
\hline \multirow{2}{*}{ Total } & Female & 69 & 74,72 & 5155,50 & \multirow[t]{2}{*}{2740,500} & \multirow[t]{2}{*}{0,648} \\
\hline & Male & 83 & 77,98 & 6472,50 & & \\
\hline
\end{tabular}

There is no statistically significant difference $(\mathrm{U}=2823,000 ; \mathrm{p}=$ 0,879>0,05) when the scores of the Authoritarian Classroom Management Profile subscale were different according to gender.

There was no statistically significant difference $(U=2665,000, p=$ $0,457>0,05)$ when the scores obtained from the subscale of the Appreciation Administrative Profile were different according to gender.

There was no statistically significant difference $(U=2787,000 ; p=$ 0,774> 0,05) when the scores from the subscale of the Adolescent Classroom Management Profile were different according to gender.

There was no statistically significant difference $(\mathrm{U}=2808,000, \mathrm{p}=$ $0,835>0,05)$ when the scores from the subscale of Classroom Management Profile were not different according to gender.

There was no statistically significant difference $(U=2740,500 ; p=$ $0,648>0,05$ ) when comparing the scores obtained from the total (general scale) according to the gender. 


\section{Discussion and Conclusion}

According to the results of the research, there was no statistically significant difference in the scores obtained from the aggregate (from the general scale) and subscales by occupation year and age. In the study conducted on 40 private and 50 public school teachers working in different schools to determine the level of classroom management skills, Özgan, Yiğit, Aydın and Küllük (2011) stated that there was no difference between teachers.

Furthermore, there is no statistically significant difference between the total scores obtained from the scale and the subscales according to the various field in the survey. When the studies were examined, Inan and Dervent (2013) found that Physical Education teachers showed democratic tendencies in classroom management. Lewis et al. (2005) found that in their research that examines class discipline strategies applied in Australia, China and Israel, the causes of students' misconduct were related to increasingly strict discipline strategies of teachers.

According to the sex, the scores obtained from the total (general scale) did not show any statistically significant difference. In the literature, Yaşar (2008) and Tortu (2012) determined that there was no significant difference in classroom management according to gender variables in their research. Ünlü (2008), Ayar and Arslan (2008), and Taflan (2007) and Kars (2007) found significant differences in classroom management by sex compared to females in their research on different subject areas. In another study, Yilmaz (2011) found that there is no significant difference in the profile of class management according to gender variable. In another study, Çiftçi (2015) found that female teachers exhibited a more authoritarian classroom management profile compared to male teachers. Also, gender was not a significant factor in classroom management profiles that were unattended, neglected, and appreciated. In the study conducted by Kurt and Ekici (2014) with 165 prospective teachers in order to analyze the disciplinary self-efficacy perceptions of the prospective teachers according to the class management profiles, it was found that the class management profiles did not show any significant difference according to both sex and general academic achievement level.

Therefore, it is suggested that this research should be carried out in future researches using qualitative research techniques.

\section{References:}

1. Aluçdibi, F., \& Ekici, G. (2012). Ortaöğretim Öğrencilerinin Biyoloji Dersi Motivasyon Düzeylerine Biyoloji Öğretmenlerinin Sınıf Yönetimi Profillerinin Etkisi. Hacettepe Üniversitesi Ë̆itim Fakültesi Dergisi, 43(43). 
2. Ayar, A. R., \& Arslan, R. (2008). İlköğretim okullarında görev yapan sınıf öğretmenlerinin "sınıf yönetimi performansının" araștırılması. Kastamonu Eğitim Dergisi, 16(2), 335-344.

3. Brown, D. F. (2005). The significance of congruent communication in effective classroom management. The Clearing House: A Journal of Educational Strategies, Issues and Ideas, 79(1), 12-15.

4. Çiftçi, A.S. (2015). İlkokul Öğretmenlerinin Sınıf Yönetim Tarzları ve Demokratik Değerlere İlişkin Görüşleri Arasındaki İlişki. DoktoraTezi, Çanakkale Onsekiz Mart Üniversitesi Eğitim Bilimleri Enstitüsü, Çanakkale.

5. Ekici, G. (2004). İlköğretim I. kademe öğretmenlerinin sınıf yönetimi profillerinin değerlendirilmesi. Ĕgitim ve Bilim, 29(131).

6. Ekici, G., \& Kurt, H. (2014). Öğretmen Adaylarının Disiplin Özyeterlik Algılarının Sınıf Yönetimi Profillerine Göre Analizi. The Analysis of Disciplinal Self-efficacy Perceptions of Student Teachers Based on Their Classroom Management Profiles.

7. Ekici, G., \& Kurt, H. (2014). Öğretmen Adaylarının Disiplin Özyeterlik Algılarının Sınıf Yönetimi Profillerine Göre Analizi The Analysis of Disciplinal Self-efficacy Perceptions of Student Teachers Based on Their Classroom Management Profiles.

8. Ingram, K., Lewis-Palmer, T., \& Sugai, G. (2005). Function-Based Intervention Planning Comparing the Effectiveness of FBA FunctionBased and Non-Function-Based Intervention Plans. Journal of Positive Behavior Interventions, 7(4), 224-236.

9. İnan, M., \& Dervent, F. (2013). Beden Eğitimi ve Sınıf Öğretmeni Adaylarının Demokratik Eğilimleri ile Sınıf Yönetimi Anlayışları Arasındaki Đlişkinin Đncelenmesi Investigation of the Relevance Between Democratic Tendencies and Classroom Management Perceptions of Preservice Physical Education and Classroom Teachers. Pegem Ĕ̈itim ve Öğretim Dergisi, 3(1), 27-34.

10. Karasar, N. (2000). Bilimsel araştırma yöntemi (17. Bask). Ankara: Nobel Yayın Dağıtım.

11. Kars, Y. E. (2007). Din kültürü ve ahlak bilgisi öğretmenlerinin kendi algılarına dayalı sınıf yönetimi yeterlikleri (Konak ilçesi örneği). Yayımlanmamış Yüksek Lisans Tezi, Dokuz Eylül Üniversitesi Sosyal Bilimler Enstitüsü, İzmir.

12. Kurt, H. (2013). Biyoloyi Öğretmenlerinin Öğrenci Başarısından Sorumluluk Algılarının Sinıf Yönetimi Profillerine Göre Analizi. Electronic Turkish Studies, 8(6).

13. Özçakır, S. (2007). Beden eğitimi öğretmenlerinin sınıf yönetimi anlayışları: Düzce ili örneği. A.Đ.B.Ü. Sosyal Bilimler Enstitüsü, 
Beden Eğitimi Öğretmenliği Anabilim Dalı, Yayınlanmamış Yüksek Lisans Tezi, Bolu.

14. Özgan, H., Yiğit, C., Aydın, Z., \& Küllük, M. C. (2010). İlköğretim okulu öğretmenlerinin sınıf yönetimine ilişkin algılarının incelenmesi ve karşıllaştırılması. Gaziantep University Journal of Social Sciences, 10(1), 615-635.

15. Sarıtaş, M. (2003). Sınıf Yönetimi ve Disiplini ile İlgili Kurallar Geliştirme. L. Küçükahmet (Editör). Sınıf Yönetiminde Yeni Yaklaşımlar (s. 45-83). Ankara: Nobel Yayınları.

16. Taflan, A. (2007). Öğretmen görüşleri ve öğrenci algısıyla sınıf yönetimi stratejilerinin değerlendirilmesi. Istanbul: Yeditepe Üniversitesi Sosyal Bilimler Enstitüsü, Yayımlanmamış Yüksek Lisans Tezi.

17. Tortu E (2012). İlköğretim Öğrencilerinin Beden Eğitimi Dersinde Sınıf Yönetimine Ait Görüşlerinin İncelenmesi. Yüksek Lisans Tezi, Kırıkkale Üniversitesi Sağlık Bilimleri Enstitüsü, Kırıkkale.

18. Ünlü, H. (2008). Beden eğitimi öğretmenlerinin yeterlikleri ve sınıf yönetimi davranışları. Doktora Tezi. Gazi Üniversitesi Eğitim Bilimleri Enstitüsü, Ankara.

19. Yaşar, S. (2008). Classroom management approaches of primary school teachers (Doctoral dissertation, Middle East Technical University).

20. Yılmaz, K. (2011). İlköğretim Okulu Öğretmenlerinin Sınıf Yönetimi Tarzları ile Demokratik Değerlere İlişkin Görüşleri Arasındaki İlişki. Journal of Values Education, 9(21). 